\title{
A Health Insurance System for Maintaining or Improving Activities of Daily Living in Acute Wards in Japan
}

\author{
Yoichiro Aoyagi ${ }^{*}$, Eiichi Saitoh ${ }^{1}$, Yuji Kono ${ }^{2}$ and Etsuko Mori²
}

${ }^{1}$ Department of Rehabilitation Medicine I, Fujita Health University, Toyoake, Japan

${ }^{2}$ Department of Rehabilitation, Fujita Health University Banbuntane Hotokukai Hospital, Nagoya, Japan

\begin{abstract}
Inpatients' physical activity is commonly restricted because of treatment, leading to declines in Activities of Daily Living (ADLs) and prolonged hospital stay. In 2014, an additional health insurance system for maintaining or improving ADLs ("ADL ijikoujoto-taiseikasan" in Japanese) was newly established by the Japanese government. The fundamental policies for this system are as follows: (1) preventing functional declines of ADLs during hospitalization and facilitating early discharge and (2) enhancing multidisciplinary approaches and safety management, preventing disuse syndrome and pressure sores, and sharing information with patients and their families. To date, two studies have reported that immediate intervention by assigned physical and/or occupational therapists in acute wards under this health insurance system effectively prevents declines in ADLs and reduces hospital stay.
\end{abstract}

Keywords: Activities of daily living; Physical activity; Elderly patient

\section{Introduction}

The Japanese society is rapidly aging. When elderly patients are admitted to an acute care hospital, their physical activities are often restricted because of treatment. Accordingly, their physical function and Activities of Daily Living (ADLs) decrease and the length of hospital stay tends to be protracted. Decreasing hospital stay has been one of the primary objectives of the Ministry of Health, Labour and Welfare's plan for reducing healthcare costs by ensuring the appropriate and efficient provision of medical services $[1,2]$. It is essential to prevent declines in ADLs through rehabilitation interventions in the early phase of hospitalization.

\section{Importance of Early Mobilization}

Early mobilization, which has been recognized as a primary component of acute-phase rehabilitation interventions, is associated with better outcomes. One randomized trial of mechanically ventilated patients reported an association between early mobilization and better outcomes, including higher rates of discharge to home, fewer days in the intensive care unit, and less hospital-acquired comorbidity, such as ventilator-associated pneumonia and intensive care unit delirium [3]. Moreover, small randomized trials also have observed better physical functional status when patients who experienced a stroke are mobilized 24-48 h after hospitalization [4]. Another large-scale clinical registry reported the association of early mobilization with clinical outcomes in patients following heart failure. Early ambulation within 2 days of hospitalization is also associated with decreased length of hospital stay and post-discharge 30-days readmission rates [5].

Elderly patient populations such as those in Japan often have multiple comorbidities and physical frailty, which may contribute to longer hospital stay and lower rates of discharge to home. Iatrogenic sarcopenia caused by excessive bed rest is one of the major hospitalacquired comorbidities in the clinical setting, and it is considered preventable by early mobilization.

\section{A New Health Insurance System to Maintain or Improve ADLs in Acute Wards in Japan}

In April 2014, an additional health insurance system aiming to maintain or improve ADLs ("ADL ijikoujoto-taiseikasan" in Japanese) was newly established by the Japanese government. The main purpose of this new system is promoting early mobilization immediately after admission in acute-phase wards to prevent functional declines because of disuse and enhance early discharge.

The main criteria for hospitals to participate in this health insurance system are as follows: (a) the percentage of patients with decreased ADLs as evaluated using the Barthel Index at discharge compared with that at admission should be less than $3 \%$ and (b) the proportion of patients with hospital-acquired pressure sores should be less than 1.5\% [6] The fundamental policies for this system are as follows: (1) preventing functional declines of ADLs during hospital stay and facilitating early discharge and (2) enhancing multidisciplinary approach and safety management, preventing disuse syndrome and pressure sores, and sharing information with patients and their families (Figure 1). The advantages of this system are both the early identification of patients requiring rehabilitation and early evaluation and intervention by fulltime physical and/or occupational therapists assigned to an acute ward of the hospital at an early stage after admission, which is arguably effective, especially for reducing the length of hospital stay. Under this system, an assigned physical therapist in an acute ward performs rehabilitative interventions $>30$ min per day for up to 14 days after admission until discharge or before the start of a conventional individual rehabilitation program.

Thirty-two acute hospitals participated in the additional health insurance system in 2015. Since then, the number of the hospitals gradually to 66 hospitals in 2017, and it is expected to further increase in the future.

\section{Impact of The Additional Health Insurance System}

To date, two studies have investigated the effects of assigning

*Corresponding author: Yoichiro Aoyagi, MD, PhD, Department of Rehabilitation Medicine, School of Medicine, Fujita Health University, 1-98 Dengakugakubo, Kutsukake, Toyoake, Aichi 470-1192, Japan, Tel: +81-562-93-2167; Fax: +90 342 47207 18; E-mail: yyy@rc5.so-net.ne.jp

Received June 21, 2018; Accepted June 27, 2018; Published June 30, 2018

Citation: Aoyagi Y, Saitoh E, Kono Y, Mori E (2018) A Health Insurance System for Maintaining or Improving Activities of Daily Living in Acute Wards in Japan. Int Neurorehabilitation 5: 324. doi: 10.4172/2376-0281.1000324

Copyright: @ 2018 Aoyagi Y, et al. This is an open-access article distributed unde the terms of the Creative Commons Attribution License, which permits unrestricted use, distribution, and reproduction in any medium, provided the original author and source are credited. 

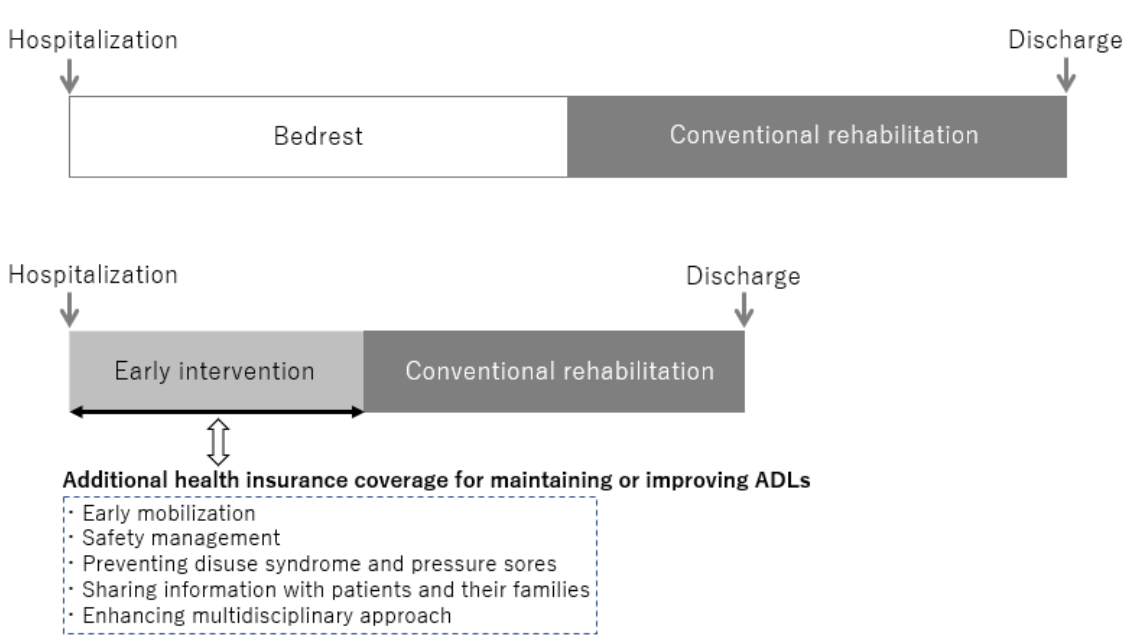

Figure 1: Potential benefits of additional health insurance coverage for Activities of Daily Living (ADLs). The upper graph represents the conventional course from hospitalization to discharge. The lower graph represents the shortened length of hospital stay achieved through early intervention by assigned therapists under the added health insurance coverage.

\begin{tabular}{|l|c|c|c|}
\hline & Author (year) & ADL's group & Control group \\
\hline \multirow{2}{*}{ Number of days before the initiation of rehabilitation } & Kono et al. [8] & $3.7 \pm 2.0$ & $11.7 \pm 6.9$ \\
\hline \multirow{2}{*}{ Length of hospital stay (days) } & Hirano et al. [7] & $5.0 \pm 4.3$ & $11.2 \pm 14.5$ \\
\hline \multirow{2}{*}{ Duration of rehabilitation intervention (days) } & Kono et al. [8] & $26.1 \pm 12.6$ & $41.8 \pm 17.1$ \\
\cline { 2 - 4 } & Hirano et al. [7] & $28.6 \pm 18.7$ & $37.5 \pm 27.3$ \\
\hline \multirow{2}{*}{ FIM score at discharge } & Kono et al. [8] & $20.3 \pm 10.4$ & $25.0 \pm 15.1$ \\
\cline { 2 - 4 } & Hirano et al. [7] & $23.6 \pm 16.2$ & $25.3 \pm 21.0$ \\
\hline
\end{tabular}

Note: n.s.- not significant

Table 1: Comparisons of patients covered under the additional insurance system and controls in prior studies.

physical and/or occupational therapists to a ward using the new additional health insurance system to maintain or improve ADLs. Hirano et al. [7] reported reductions in the number of days before the initiation of individual rehabilitation and the length of hospital stay in a respiratory medicine ward (Table 1). Our current study conducted in a cardiology ward [8] revealed that the number of days before the initiation of conventional individual rehabilitation program, duration of individual rehabilitation programs, and length of hospital stay were decreased after implementation of this new system, whereas the Functional Independence Measure (FIM) level at discharge was maintained, indicating that the preferred outcomes were attained with a shorter length of hospital stay. Our study corroborated the findings of the prior study by Hirano et al. [7]. The underlying factors of this desirable outcome may be attributed to the prevention of declines in ADLs at the initiation of rehabilitative interventions through assigning therapists to a ward and performing early mobilization.

In a conventional rehabilitation program, physical and/or occupational therapy interventions are provided if ordered by an attending physician or physiatrist. One feature of the new system is that direct preventive intervention by assigned therapists becomes available in the early stage of hospitalization. Decreases in the time before the initiation of conventional rehabilitation, duration of rehabilitation, and length of hospital stay were possibly attained by selecting patients with high risks of $\mathrm{ADL}$ declines by identifying the disease state and $\mathrm{ADL}$ levels of patients at an early stage after admission and connecting these patients to disease-specific rehabilitation programs immediately.
Taking together, the recent evidence leads us to conclude that immediate intervention by assigned physical and/or occupational therapists in acute wards under the new health insurance system effectively prevents declines of ADLs and reduces the length of hospital stay.

\section{References}

1. Health Insurance Bureau-Ministry of Health (2014) Labour and Welfare: Moderation in healthcare cost.

2. National Federation of Health Insurance Societies (2011) Investigation research on functional classification of acute care and acute hospital.

3. Schweickert WD, Pohlman MC, Pohlman AS, Nigos C, Pawlik AJ, et al. (2009) Early physical and occupational therapy in mechanically ventilated, critically ill patients: A randomised controlled trial. Lancet 373: 1874-1882.

4. Cumming TB, Thrift AG, Collier JM, Churilov L, Dewey HM, et al. (2011) Very early mobilization after stroke fast-tracks return to walking: Further results from the phase II AVERT randomized controlled trial. Stroke 42: 153-158.

5. Fleming LM, Zhao X, DeVore AD, Heidenreich PA, Yancy CW, et al. (2018) Early ambulation among hospitalized heart failure patients is associated with reduced length of stay and 30-day readmissions. Circ Heart Fail 11: e004634.

6. Hiroshi U (2014) Outline of medical treatment fee revision in Heisei 20 year. Ministry of Health, Labor and Welfare.

7. Hirano A, Kato M, Fujimura K, Hayakawa M, Kagaya H, et al. (2016) Assignment of physical therapists in acute hospital: Approach for extra medica bills to maintain or improve ADL in respiratory medicine ward. J Jpn Phys Ther Assoc 43: 255-262.

8. Kono Y, Aoyagi Y, Kayukawa T, Mori E, Ishikawa A, et al. (2017) Efforts and effects of additional medical coverage to maintain or improve activities of daily living in an acute cardiovascular internal medicine ward. Jpn J Compr Rehabil Sci 8: 104-108. 